\title{
Follow-up optimized phononic band structure design based on reserved inclusions
}

\author{
Kai Wanga, Ying Liu ${ }^{b^{*}}$, Bin Wang \\ ${ }^{a}$ Department of Mechanics, School of Civil Engineering, Beijing Jiaotong University, Beijing 100044 \\ PR China \\ ${ }^{\mathrm{b}}$ School of civil Engineering, Beijing Jiaotong University, Beijing 100044 PR China \\ Email: yliu5@bjtu.edu.cn; Tel: 86-10-51688763; Fax: 86-10-51682094 \\ ${ }^{\mathrm{c}}$ Department of Mechanics, Aerospace and Civil Engineering, Brunel University, London Kingston Lane, \\ Uxbridge Middlesex UB8 3PH, United Kingdom
}

For functional demands, inclusions are always reserved beforehand in a structural design. In this paper, a follow-up optimized phononic band structure design is performed based on a 2D structure with reserved inclusions but no absolute band gaps for the purpose to simultaneously achieve acoustic abatement except for functional demands. Firstly, the strategy to optimize the band structure based on bidirectional evolutionary structural optimization algorithm is proposed. Then the effects of geometrical or physical parameters on the final optimization results are discussed. Numerical results show that there is a material gravitation phenomenon around the inclusions in the topological optimization. Factors affecting the material gravitation are discussed in details. This work will lead an important guidance in the multifunctional design of multi-phase phononic crystals.

Keywords: Phononic crystals; Topology optimization; Absolute band gap; Material gravitation phenomenon 


\section{Introduction}

Along with the development of electronic packaging and flexible wearable technology, the multifunctional dynamic design of apparatus in the limited space becomes more and more crucial. For certain apparatuses, reserved inclusions are periodically distributed for functional demands. In a system with periodical distributed two or more phases there may exist absolute band gaps (ABGs) within which the propagation of elastic waves is restrained. This distinct property provides an effective strategy in vibration suppression, which motivates the idea to further make band gap optimization based on a structure with beforehand periodically reserved inclusions but initially no ABG existence.

Since the firstly proposal of the idea in 1993 [1], amounts of researches have been undertaken to explore the theoretical concept of phononic crystals (PnCs), in which there are certain frequency bands within which the elastic waves are forbidden to propagate. Liu et al. [2] fabricated PnCs and found the ABGs based on a localized resonant mechanism. Zhao et al. [3] studied the peculiar transmission peaks of PnCs in both theoretical analysis and experimental observation. Moreover, band gaps in PnCs with one [4-7], two [8-14] or three [15-18] dimensional lattices were widely studied. Along with the mature of $\mathrm{ABG}$ determination in given PnCs, PnC optimization according to application demands becomes essential. Considerable interests have been devoted to design PnCs with larger ABGs based on material design [19-22] and structural schemes [23-25]. Actually, topology optimization is an effective tool in PnC ABG design. Sigmund and Jensen [26] firstly introduce solid isotropic material penalization method (SIMP) and finite element method (FEM) in PnC ABG design. Following their work, Hussein et al. [27] presented a design approach based on a multi objective genetic algorithm (GA) to optimize one-dimensional PnCs in layered materials. Dong et al. [28] proposed a 'coarse to fine' two-stage genetic algorithm to optimize ABGs in 2D solid-solid binary-component PnCs. Due to the complicated solution space in GA method, the optimal design of 
PnCs usually needs over thousands of evolutions. Toward an efficient and reliable optimization of ABGs, bidirectional evolutionary structural optimization algorithm (BESO) [29] was firstly introduced in the design of PnCs in 2016. Following their work, Li et al. [30] proposed a BESO design for in-plane ABG in the structures consisting of hollow rods and circular solid. Zhang et al. [31] performed the topology optimization for ABGs in PnCs with sixfold symmetric hexagonal lattice. Considering a relatively high stiffness of PnCs, Li et al. [32] conducted optimization of cellular PnCs to simultaneously maximize band gap size, and bulk or shear modulus under prescribed filling fractions.

It is seen that numerous works have been done to obtain optimized ABGs. In real application, there exist the situation that reserved inclusions are periodically arranged in the space. If through certain ABG optimization based on the reserved periodical structures, it is possible to simultaneously realize vibration suppression except for functional demands. Motivated by this idea, the follow-up ABG optimization design with reserved inclusions is proposed. Based on the BESO method, the algorithm is firstly established for topological optimization of ABGs in structures with periodically reserved inclusions. The topological optimization design for binary-component solid PnCs, binary-component porous PnCs, and ternary-component solid PnCs is performed. The effects of material and geometrical parameters on ABGs are clarified in detail. At last, the conclusion is given.

\section{Description of the problem}

A typical representation of a 2D periodical reserved structure with a square inclusion is shown in Fig.1. Orange area represents the reserved inclusion (denoted by A), meanwhile blue area is the matrix material (denoted by B). Since it is squarely periodically distributed in 2D space, a unit cell with the length $\boldsymbol{a}$ is chosen, which is marked out by dark grey. The detailed structure of the unit cell is given in Fig. 1b. The inclusion is surrounded by light grey isolation strips with width $c$. An isolation ratio 


$$
\mathrm{R}_{c}=\frac{c}{a},
$$

is defined to indicate the strip width. The optimization is performed in the design area.

In the following study, for a certain unit cell length $a$, the filling material, $\mathrm{Pb}$, is distributed optimally in the matrix, epoxy (for dark gray design area). The effect of isolation ratio $\mathrm{R}_{c}$ on the filling material distribution and $\mathrm{ABG}$ structures is discussed. Moreover, different materials are used for inclusions and isolation strips to see the influence of acoustic mismatch between different parts. For clarity, an A-B PnC is used to represent a PnC with material A for inclusion and material B for isolation strip.

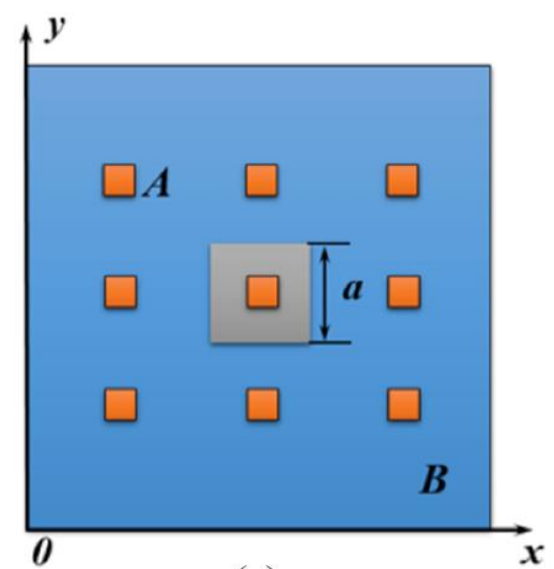

(a)

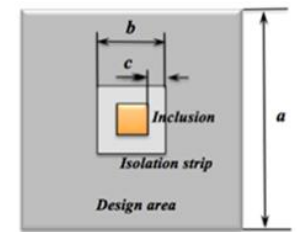

(b)

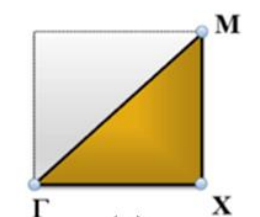

(c)

Fig. 1 Diagrammatic sketch of (a) a representative 2D inclusion phononic crystal; (b) the unit cell, and (c) the first Brillouin zone of a 2D square lattice

\section{Theory}

As shown in Fig. 1, by now, the structure with periodically reserved inclusions is transferred to a PnC. Since the unit cell is squarely distributed, the dispersion relation is obtained by wave vector sweeping the edges of the first irreducible Brillouin zone ГXM, which is plotted in Fig. 1c.

For a 2D PnC system, considering that elastic waves propagate only in the xoy plane, the wave equations are given as 


$$
-\rho(\mathbf{r}) \omega^{2} u_{i}=\nabla \cdot\left[\mu(\mathbf{r}) \nabla u_{i}\right]+\nabla \cdot\left[\mu(\mathbf{r}) \frac{\partial}{\partial x_{i}} \mathbf{u}\right]+\frac{\partial}{\partial x_{i}}[\lambda(\mathbf{r}) \nabla \cdot \mathbf{u}] \quad(i=x, y) .
$$

Here, $\mathbf{u}=\left(u_{x}, u_{y}\right)$ is the displacement vector in the transverse plane, $\mathbf{r}$ is the position vector. $\lambda$ and $\mu$ are the Lamé constant and shear modulus. $\omega$ is the angular frequency, $\rho$ is the mass density, $\nabla=(\partial x, \partial y)$ is $2 \mathrm{D}$ vector differential operator.

According to Bloch's theorem [33], the displacement vector $\mathbf{u}$ can be written as

$$
\mathbf{u}(\mathbf{r})=e^{i(\mathbf{k} \cdot \mathbf{r})} \mathbf{u}_{k}(\mathbf{r})
$$

where $\mathbf{k}=\left(k_{x}, k_{y}\right)$ is the wave vector limited to the first Brillouin zone of the reciprocal lattice as shown in Fig. 1. $\mathbf{u}_{k}(\mathbf{r})$ is a periodical vector function with the same PnC lattice periodicity. $i$ is the imaginary unit.

In the present work, the finite element method (FEM) is applied to calculate the band structures of the proposed PnCs. The discrete form of eigenvalue equations in a unit cell is written as

$$
\left(\mathbf{K}-\omega^{2} \mathbf{M}\right) \mathbf{U}=0
$$

where $\mathbf{K}$ is the global stiffness matrix of the unit cell, $\mathbf{U}$ is the nodes displacement, $\mathbf{M}$ is the global mass matrix. The Bloch conditions, Eq. (3), should be applied on the boundaries of the unit cell, the displacement field then can be expressed as

$$
\mathbf{U}(\mathbf{r}+\mathbf{a})=e^{i(\mathbf{k} \cdot \mathbf{a})} \mathbf{U}(\mathbf{r})
$$

where $\mathbf{a}$ is the lattice basis vector that generates the point lattice associated with the PnCs. $\mathbf{r}$ is the position vector located at the boundary nodes. COMSOL with MATLAB is used here to solve the eigenvalues of Eq. (5) under the complex boundary condition of Eq (5). The model built in COMSOL is saved as a MATLAB-compatible '.m' file. The unit cell is meshed by triangular Lagrange quadratic elements provided by the code. Eigen-frequency analysis is selected and the direct SParse Object Oriented Linear Equations Solver (SPOOLES) is chosen as the solver. The 
proposed '.m' file is programmed to let the wave vector $\mathbf{k}$ sweep the edges of the irreducible Brillouin zone as shown in Fig. 1. Then the whole dispersion relations are obtained.

\section{BESO optimization}

BESO method is a high-efficiency topology optimization method based on the material removal and addition scheme to determine the optimized structure. Staring from given inclusions and isolation strips, BESO will evolve the unit cells to the optimal ones based on sensitivity analyses in each evolution. The optimization goal is to open/maximize ABGs in PnCs with reserved inclusions. The objective function is

$$
\text { Maximize }: f\left(\sum\right)=2 * \frac{\min _{\mathbf{k}}: \omega_{n+1}\left(\sum, \mathbf{k}\right)-\max _{\mathbf{k}}: \omega_{n}\left(\sum, \mathbf{k}\right)}{\min _{\mathbf{k}}: \omega_{n+1}\left(\sum, \mathbf{k}\right)+\max _{\mathbf{k}}: \omega_{n}\left(\sum, \mathbf{k}\right)},
$$

where $f$ is the relative bandgap width between the $n^{\text {th }}$ and $n+1^{\text {th }}$ bands given by the relative ratio of the gap width and the central frequency. $n$ is the serial number of the energy band. $\sum$ denotes topology of the PnC unit cell. The unit cell is divided into $\mathrm{N} \times \mathrm{N}$ square pixels. Design variable $x_{i}\left(i=1,2,3 \ldots \mathrm{N}^{2}\right)$ for each pixel is defined to describe the material distribution. In the present calculation, the square unit cell is discretized into $25 \times 254$-node quadrilateral pixels. When the reserved inclusion is given, in the following optimization, only the material type of design area needs to be determined. The value 1 or 0 for the pixel in design area means that the pixel is made from filling material $(\mathrm{Pb})$ or matrix material (epoxy), respectively. To simplify the calculation, considering the square-symmetry of the unit cell, only one-eighth of the unit cell really needs to be considered.

The volume fraction of the filling material $(\mathrm{Pb})$ is selected as the constraint function in the whole optimization, that is,

$$
\frac{\sum_{i=1}^{\mathrm{T}} v_{i} x_{i}}{\mathrm{~V}^{\mathrm{T}}}=\mathrm{V}_{f}^{*},\left(x_{i}=0 \text { or } 1\right)
$$


where $\mathrm{T}$ is the total number of square pixels which in one-unit cell. $v_{i}$ denotes the volume of element $i, \mathrm{~V}_{f}^{*}$ is the prescribed volume of filling material $(\mathrm{Pb}), \mathrm{V}^{\mathrm{T}}$ is the total volume of square pixels in in one-unit cell. Volume fraction of the filling material starts from a relative small initial fraction $\mathrm{V}_{i}^{*}$ and gradually increases to the prescribed volume fraction $\mathrm{V}_{f}^{*}$ following

$$
\mathrm{V}_{p+1}=\mathrm{V}_{p}(1+E R) \text { when } \mathrm{V}_{p+1} \leq \mathrm{V}_{f}^{*}(p=1,2,3 \ldots)
$$

where $\mathrm{ER}$ is the evolution rate and $p$ is the iteration number. $\mathrm{V}_{p}$ refers to the volume fraction of filling material in the $p^{\text {th }}$ iteration, which will keep constant once it has achieved the specified volume fraction $\mathrm{V}_{f}^{*}$.

The removal and addition of material in BESO scheme is based on the sensitivity number ranking of the pixels made from matrix material and filling material together. Depending on the sensitivity ranking, the value of $x_{i}$ will be set to 1 or 0 to realize the volume evolution of filling material which is shown in Eq. (8). The calculation of the initial sensitivity numbers with $x_{i}=0$ (matrix material) can be expressed as

$$
\alpha_{i}^{e}=\frac{\partial f}{\partial \sum_{i}}=\frac{\partial f\left(+\Delta \sum_{i}\right)-f(\Sigma)}{\partial \sum_{i}}
$$

where $\sum=\left\{x_{1}, x_{2}, \ldots, x_{p} \ldots, x_{n}\right\}^{\mathrm{T}}$ is the proposed topological distribution of the unit cell, $\Delta \sum=\left\{0,0, \ldots, 0+\Delta x_{i} \ldots\right.$, $0\}^{\mathrm{T}}$ is a disturbance of $\Delta x_{i}$ on the $i^{t h}$ design. The disturbance $\Delta \sum$ is defined by the model module in FEM program, then the sensitivity number of the $i^{\text {th }}$ pixel with $x_{\mathrm{i}}=0$ can be obtained by the FE bandgap calculation which is mentioned in section 3 and Eq. (6). Sensitivity numbers for the case of $x_{\mathrm{i}}=1$ (filling material) is extrapolated by the following filter scheme[34].

Firstly, nodal sensitivity numbers for pixels with $x_{i}=0$ (matrix material) is calculated as

$$
\alpha_{i}^{\mathrm{n}}=\sum_{j=1}^{\mathrm{M}} w_{j} \alpha_{j}^{e}
$$

where $\mathrm{M}$ is the total number of pixels with $x_{p}=0$ connected to the $i^{\text {th }}$ pixel. $\alpha_{j}^{e}$ is the initial sensitive number calculated by Eq. (9). $w_{j}$ is the weight factor of the $j^{\text {th }}$ pixel given as 


$$
w_{j}=\frac{1}{\mathrm{M}-1}\left(1-\frac{r_{i j}}{\sum_{j=1}^{\mathrm{M}} r_{i j}}\right),
$$

where $r_{i j}$ is the center distance between the pixel $i$ and pixel $j$. Then the modified sensitivity number of the $i^{\text {th }}$ pixel are determined by

$$
\alpha_{i}^{m}=\frac{\sum_{j=1}^{\mathrm{M}} w\left(r_{i j}\right) \alpha_{j}^{\mathrm{n}}}{\sum_{j=1}^{\mathrm{M}} w\left(r_{i j}\right)},
$$

where $\alpha_{j}^{\mathrm{n}}$ is the nodal sensitivity number obtained by Eq. (10). $w\left(r_{i j}\right)$ is the linear weight factor given as

$$
w\left(r_{i j}\right)=\left\{\begin{array}{cl}
r_{\min }-r_{i j}, & \text { for } r_{i j} \leq r_{\min } \\
0 & \text { for } r_{i j}>r_{\text {min }}
\end{array}\right.
$$

where $r_{\min }$ is the filter radius. Considering the convergence of the solution, the modified sensitivity number is further averaged by its historical information after the first iteration [35], that is,

$$
\alpha_{i, p}=\frac{1}{2}\left(\alpha_{i, p}^{m}+\alpha_{i, p-1}\right)
$$

where $p$ is the current iteration number, $\alpha_{i, p}^{m}$ is the modified sensitivity number of the pixel $i$ in the $p^{\text {th }}$ iteration obtained from Eq. (12). The design variable $x_{i}$ is updated to 1 and 0 based on the relative ranking of the final sensitivity number $\tilde{\alpha}_{i, p}$ to realize the volume evolution until the convergence criterion is satisfied, which is defined as:

$$
\text { error }=\frac{\left|\sum_{i=1}^{\mathrm{N}} f_{p-i+1}-f_{p-\mathrm{N}-i+1}\right|}{\sum_{i=1}^{\mathrm{N}} f_{p-i+1}} \leq \tau,
$$

where $f_{p}$ is the objective function in $p^{\text {th }}$ iteration, $\tau=0.1$ is an allowable convergence tolerance. $\mathrm{N}$ is an integer number. In this paper, $\mathrm{N}$ is defined to be 3 since the change in the mean compliance is acceptably small over the last 6 iterations.

The BESO procedure used to perform the PnC topological optimization is described as 
1. Define BESO parameters, including the objective function, the objective volume of filling material, filter radius, evolutionary ratio ER, and allowable convergence tolerance;

2. Establish the initial model with given inclusions, isolation strips and design area based on $\mathrm{N} \times \mathrm{N}$ square pixels;

3. Conduct the FE band structure calculation;

4. Calculate, filter and average the sensitivity numbers;

5. Determine the volume fraction of filling material according Eq. (8);

6. Reset the design variable $x_{i}$ of elements in the design area to construct a new design according to sensitivity numbers and the volume fraction;

7. Repeat steps 3 to 6 until the constraint volume is achieved and the convergence criterion is satisfied.

\section{Results and discussion}

\subsection{Phononic crystals with square scatters}

Firstly, we give the variation of the relative band width in a $\mathrm{Pb} / E$ poxy $\mathrm{PnC}$ with respect to the length size ratio of square scatter and unit cell, $a_{1} / a$. The relative band width is obtained according to Eq. (5). Matrix material is Epoxy with $\rho_{1}=1.18 \mathrm{~g} / \mathrm{cm}^{3}, \lambda_{1}=4.43 \mathrm{GPa}, \mu_{1}=1.59 \mathrm{GPa}$; meanwhile inclusion material is $\mathrm{Pb}$ with $\rho_{2}=11.6 \mathrm{~g} / \mathrm{cm}^{3}, \lambda_{2}=42.03$ $\mathrm{GPa}$, and $\mu_{2}=14.9 \mathrm{GPa}$. If the value of the relative band width is larger than zero, the ABG opens.

As shown in Fig. 2, when the inclusion is small, see, around $a_{1} / a<0.3$, the ABG could not be opened. When the inclusion size is bigger enough, an ABG is opened at $3^{\text {rd }}$ to $4^{\text {th }}$ bands. As the increase of $a_{1} / a$, the band width is increased and then gradually decreased and finally closed after $a_{1} / a>0.6$.

In the following discussion, we have $a_{1} / a=0.2$ at which no ABG opens. From this initial reserved design, we perform the structure optimization to find ABGs. 


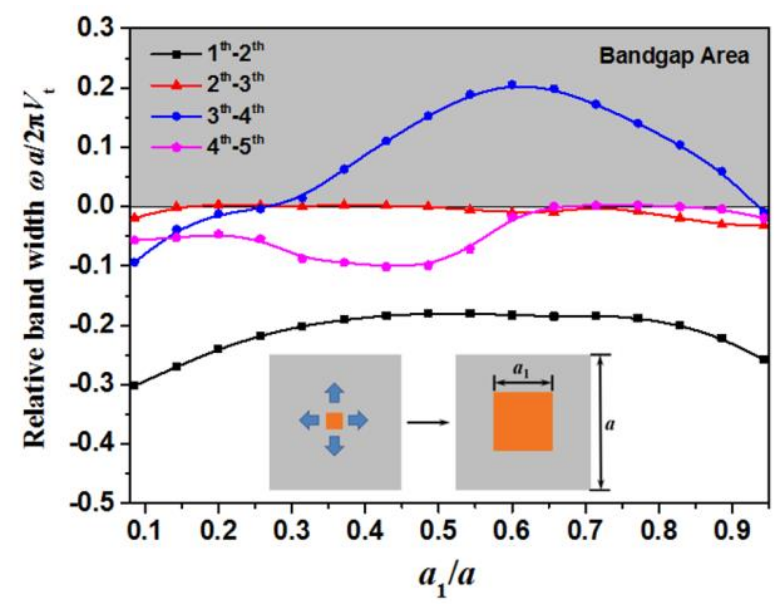

Fig. 2 Variation of the relative band width with respect to the length ratio of the scatter and the unit cell.

\subsection{Topological optimization design for PnCs with reserved inclusions}

In this section, we conduct the topological optimization design for PnCs with reserved inclusions based on the proposed BESO method. For comparison, the fill material $(\mathrm{Pb})$ and the matrix material (Epoxy) will keep unchanged. The volume fraction for filling material is set as $\mathrm{V}_{f}^{*}=0.13$.

\subsubsection{Pb-Epoxy PnCs}

Here, the material of the inclusion is $\mathrm{Pb}$ and the material in the isolation strip area is Epoxy. Filling material is $\mathrm{Pb}$.

By changing $R_{c}$, Fig. 3 shows the resulted optimized unit cells with $3 \times 3$ lattice and the corresponding band structures.

When $\mathbf{R}_{c}=0$ (Fig. 3a), which means that there is no isolation area around inclusions, the filling area is completely connected with inclusions and the optimized scatter likes a clover. The bandwidth of this optimized unit cell is 0.302 . When $\mathbf{R}_{c}=0.08$ as shown in Fig. $3 \mathrm{c}$, due to the existence of isolation area, the square inclusion is successfully 
isolated, and the filling material distributed around the isolation area to obtain the optimized bandgap 0.196. The optimized structure is four trapezoids with lower line being the length of isolation strip. Along with the increase of $\mathrm{R}_{c}$ to 0.12 , as shown in Fig. 3e, the height of the trapezoid is decreased with narrower ABG. The width of the ABG is decreased from 0.196 at $\mathbf{R}_{c}=0.08$ to 0.1 at $\mathbf{R}_{c}=0.12$. When $\mathbf{R}_{c}$ increase to 0.16 as shown in Fig. $3 \mathrm{~g}$, due to the relative wider of the isolation strip, the filling material is no longer distributed around the inclusion, but concentrated at the center of the square lattice and displays clover shape. At this time, the ABG is closed. The optimization results show that if the isolate strip is small, the filling material will be 'attracted' by the inclusion, and distributed around the inclusion. If the isolate strip is larger than a certain value, the inclusion gravitation is disappeared and the materials concentrate at the center of the square lattice.
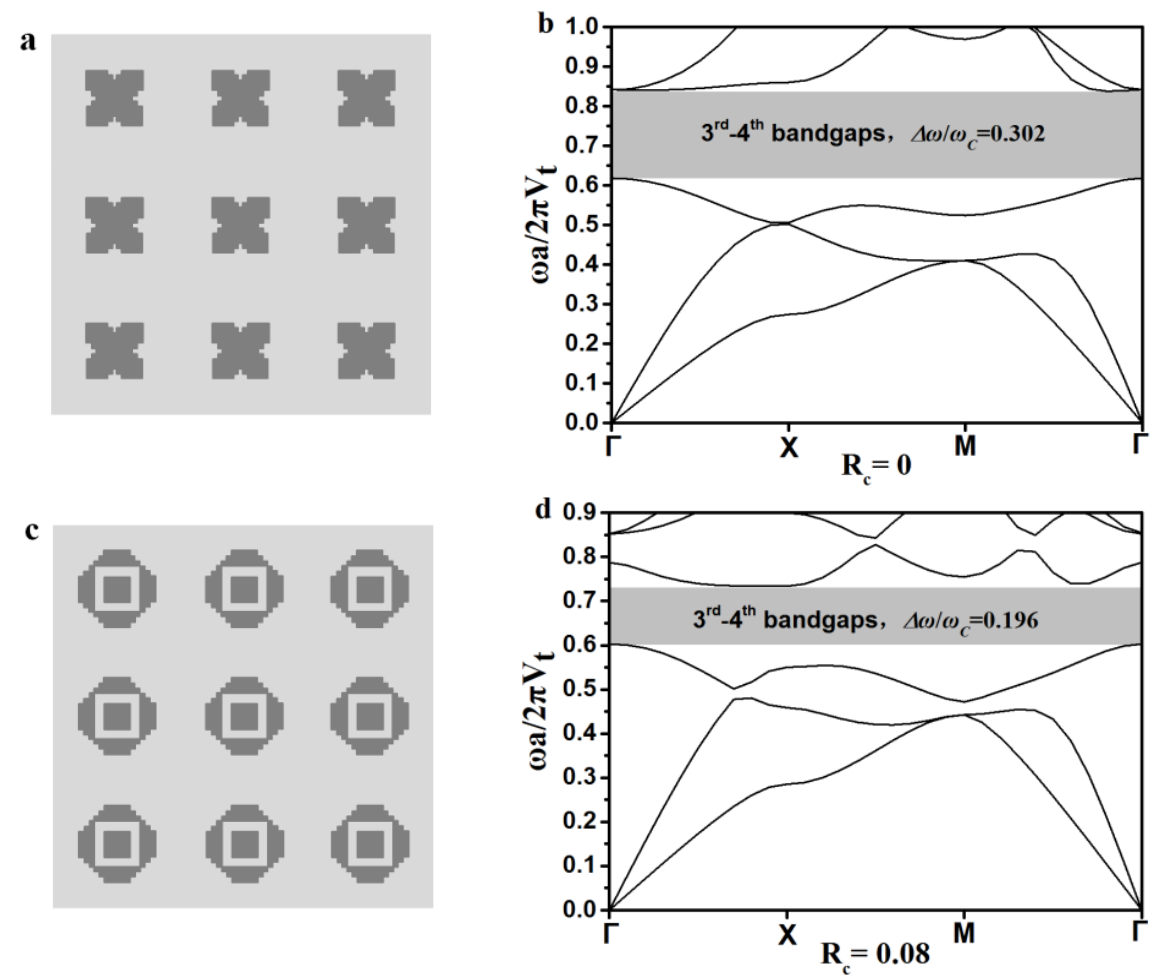

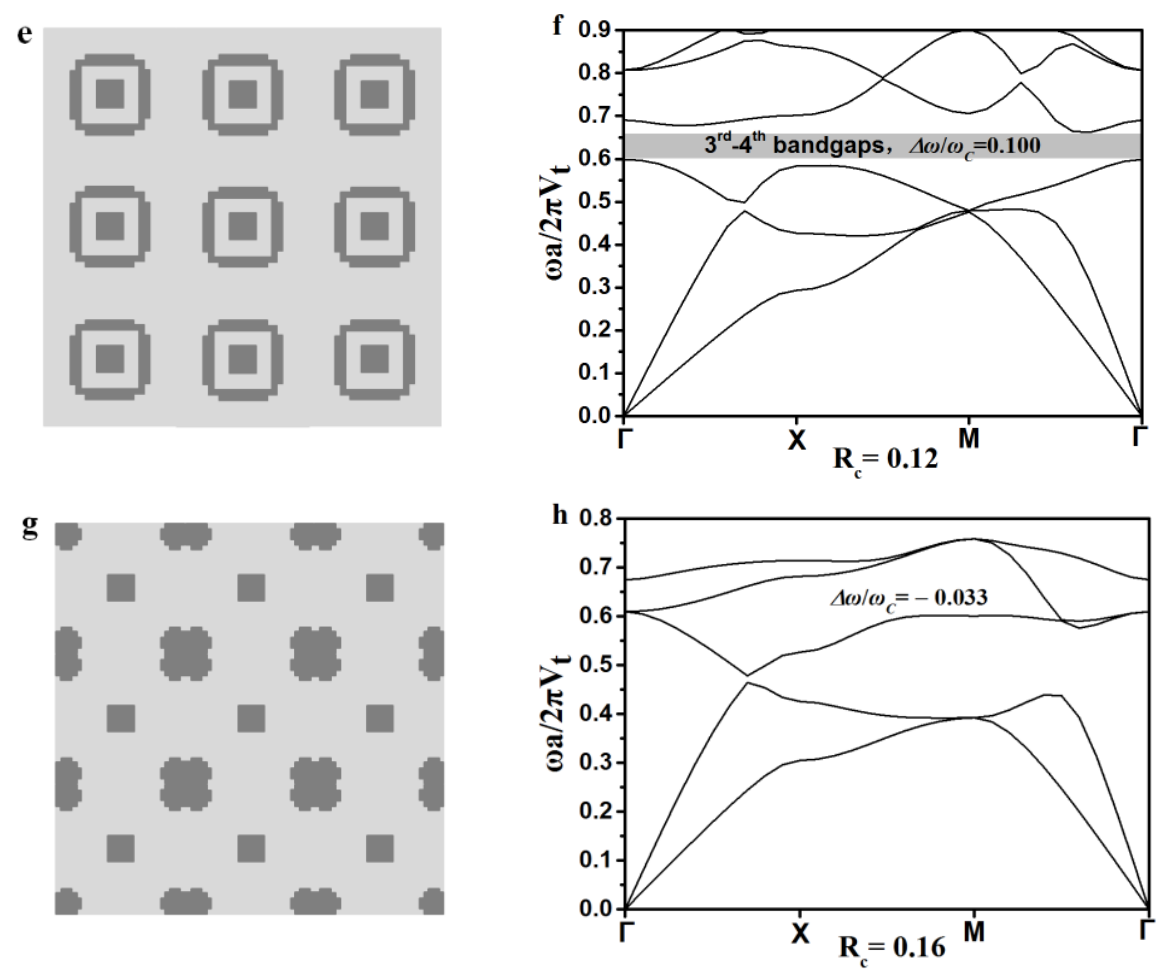

Fig.3. Optimized structures of Pb-Epoxy PnCs and the corresponding band structures for the $3^{\text {rd }}-4^{\text {th }} \mathrm{ABG}$ with different $\mathrm{R}_{c}$

\subsubsection{Au-Epoxy PnCs}

In order to see the influence of the inclusion material on the optimized topology structure, in this section, the inclusion material is changed to $\mathrm{Au}$. The other conditions are the same as those used in section 5.2.1. Compared with $\mathrm{Pb}, \mathrm{Au}$ has a higher density with the physical properties: $\rho=19.5 \mathrm{~g} / \mathrm{cm}^{3}, \lambda=160.3 \mathrm{GPa} ; \mu=29.9 \mathrm{GPa}$. Fig. 4 shows the optimized unit cells of $3 \times 3$ lattice of $\mathrm{Au}-\mathrm{Pb} \mathrm{PnCs}$ and the corresponding band structures at different isolation ratio $\mathbf{R}_{c}$.

As shown in Fig.4, Au inclusions are marked out by orange color. Comparison with Fig. 3 shows that along with the increase of the isolation strip width, the optimized filling pattern are almost the same. When there is no isolation area around $\mathrm{Au}$ inclusion, the corresponding optimized band gap size is 0.298 , a little smaller than that when the 
inclusion material is $\mathrm{Pb}$. Different from $\mathrm{Pb}$ case, there will be one more $\mathrm{ABG}$ opened between $2^{\text {nd }}$ to $3^{\text {rd }}$ bands with the bandwidth 0.065 . When $\mathrm{R}_{c}=0.08$ as shown in Fig. $4 \mathrm{c}$, the ABGs width are 0.214 which is a little larger than PnCs with $\mathrm{Pb}$ inclusions. Along with the further increasing of $\mathrm{R}_{c}$ to 0.12 and 0.16 as shown in Fig. $4 \mathrm{e}$ and $\mathrm{g}$, the ABGs width are 0.214 and 0.111 respectively. Moreover, compared with PnCs with $\mathrm{Pb}$ inclusions, the $2^{\text {nd }}-3^{\text {rd }} A B G s$ are opened with the bandwidth 0.035 and 0.025 respectively. Moreover, when $\mathrm{R}_{c}=0.16$, the filling material still distributes around the inclusion and BESO algorithm successfully finds the ABG. The results show that material with higher acoustic impedance has higher 'attraction' for filling materials.
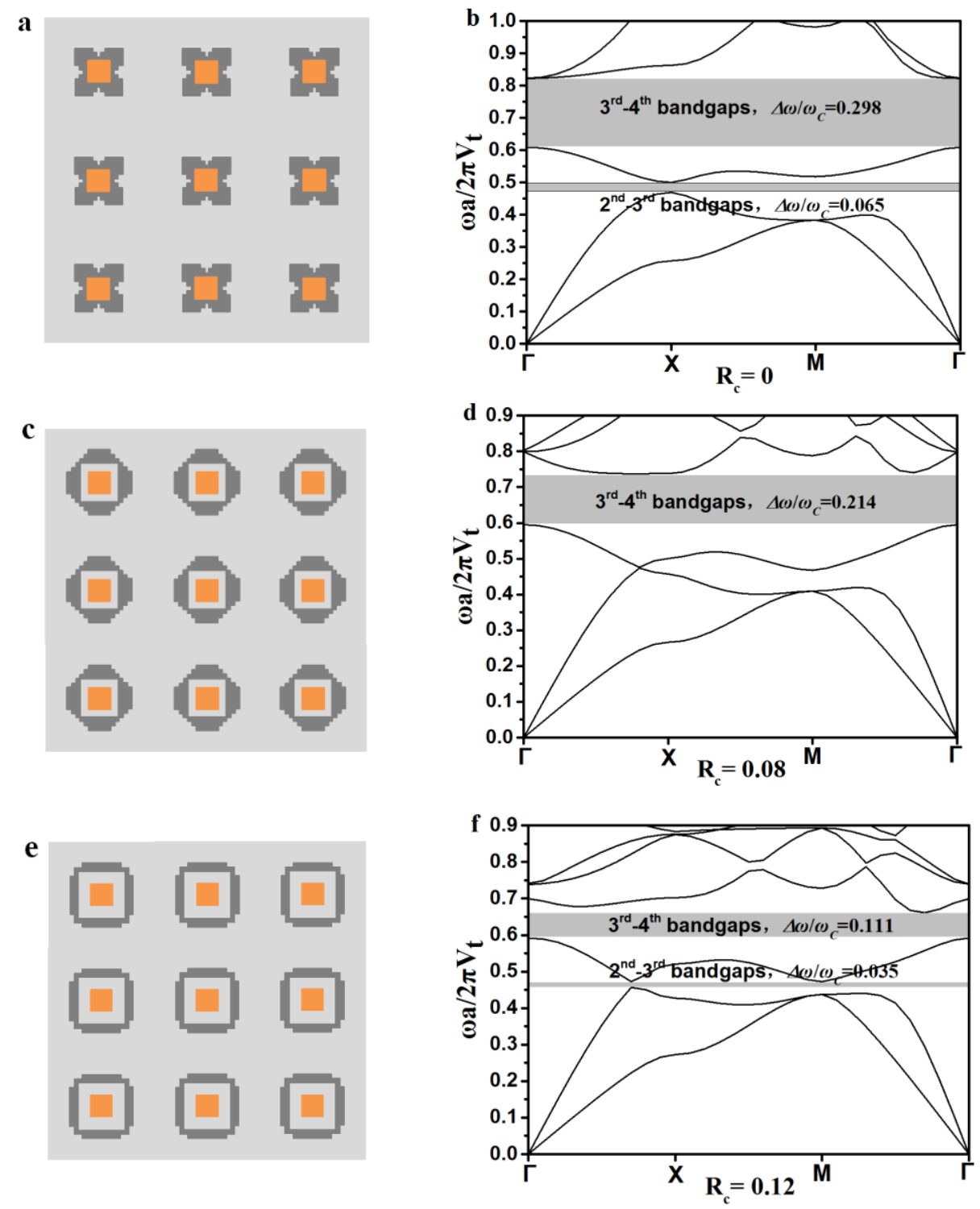

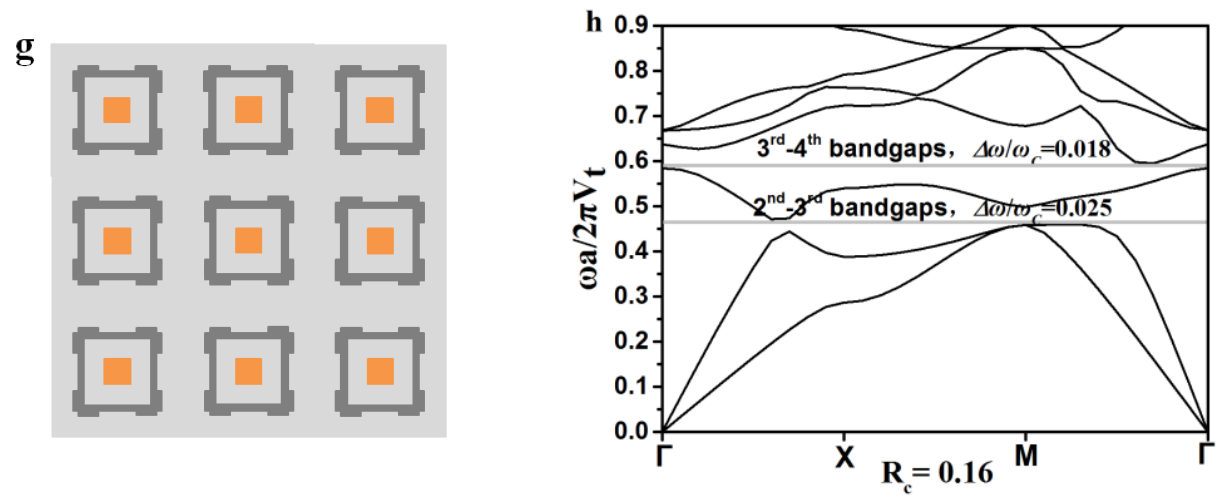

Fig.4. Optimized structures of Au-Epoxy PnCs and the corresponding band structures for the $3^{\text {rd }}-4^{\text {th }} A B G$ at different $\mathrm{R}_{c}$.

\subsubsection{Hole-Epoxy PnCs}

In order to further manifest the influence of inclusion material on the optimization result, an optimization calculation for a hole-Epoxy $\mathrm{PnC}$ is performed. In the calculation we have $a_{1} / a=0.12$. As shown in Fig. 5, along with the variation of $\mathbf{R}_{c}$ from 0 to 0.16 , the resulted optimized structure is the same. There is no material concentration around the hole. The filling material distributes at the square lattice center. The filling patter is square with semicircle at the four corners. The $3^{\text {rd }}-4^{\text {th }} \mathrm{ABG}$ width is 0.177 , for the optimized PnC, $\mathrm{ABG}$ between $2^{\text {nd }}$ to $3^{\text {rd }}$ is also opened with bandwidth 0.027 . 

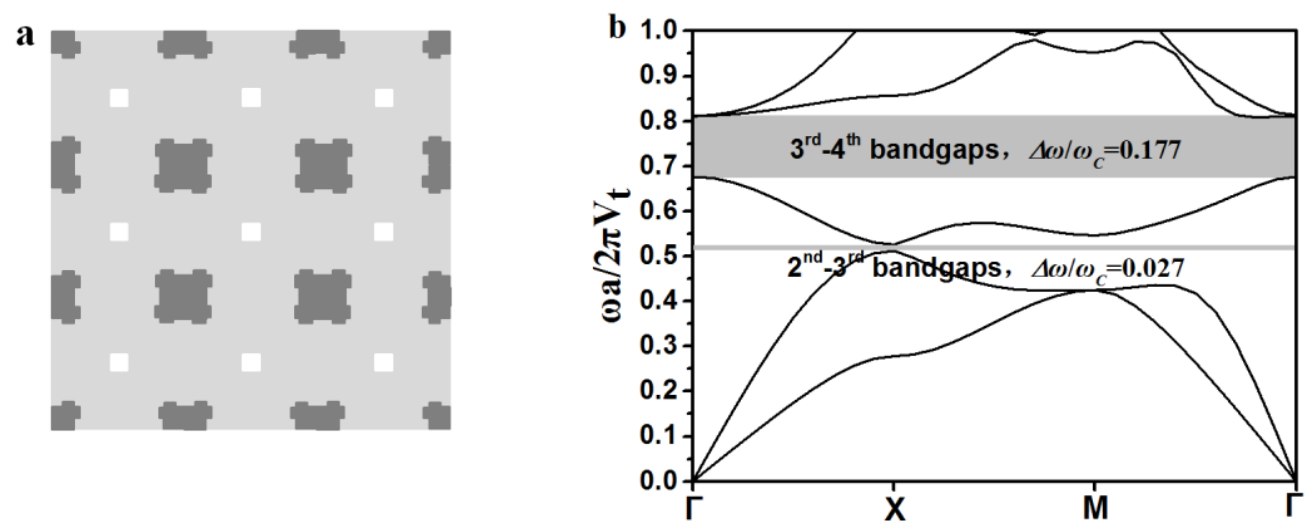

Fig.5. Optimized structures of Holes-Epoxy PnCs and the corresponding band structures for the $3^{\text {rd }}-4^{\text {th }} A B G$.

\subsection{4. $\mathrm{Pb}-\mathrm{Au} \mathrm{PnCs}$}

In order to see the effect of the isolate strip, $\mathrm{Pb}-\mathrm{Au}$ PnCs is discussed in this section. As shown in Fig. 6, due to the addition of $\mathrm{Au}$ material into the isolation area, inclusion and isolate strip combine together acting as a larger inclusion, which results in a stronger gravitation. The filling material distributes closely around the inclusion. Along with the increase of the isolate strip width, the $\mathrm{ABG}$ width is also increased. The resulted width of the $\mathrm{ABGs}$ for $\mathrm{R}_{c}$ $=0,0.08,0.12$ and 0.16 are $0.302,0.584,0.715$ and 0.774 , respectively. They are all far larger than the normal optimization design of PnCs with inclusions under the same condition.

$\mathbf{a}$

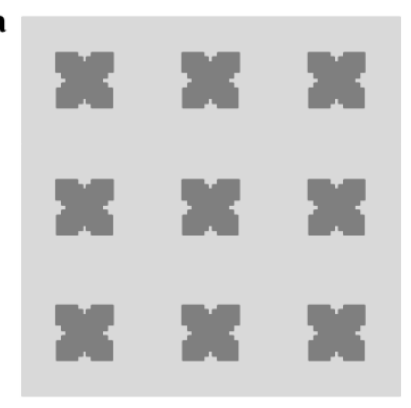

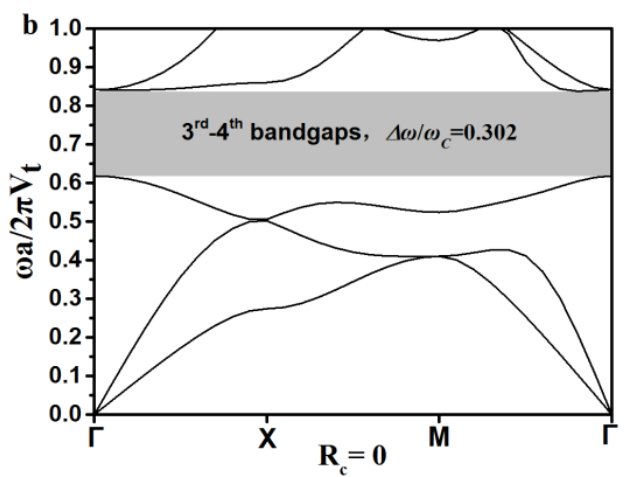



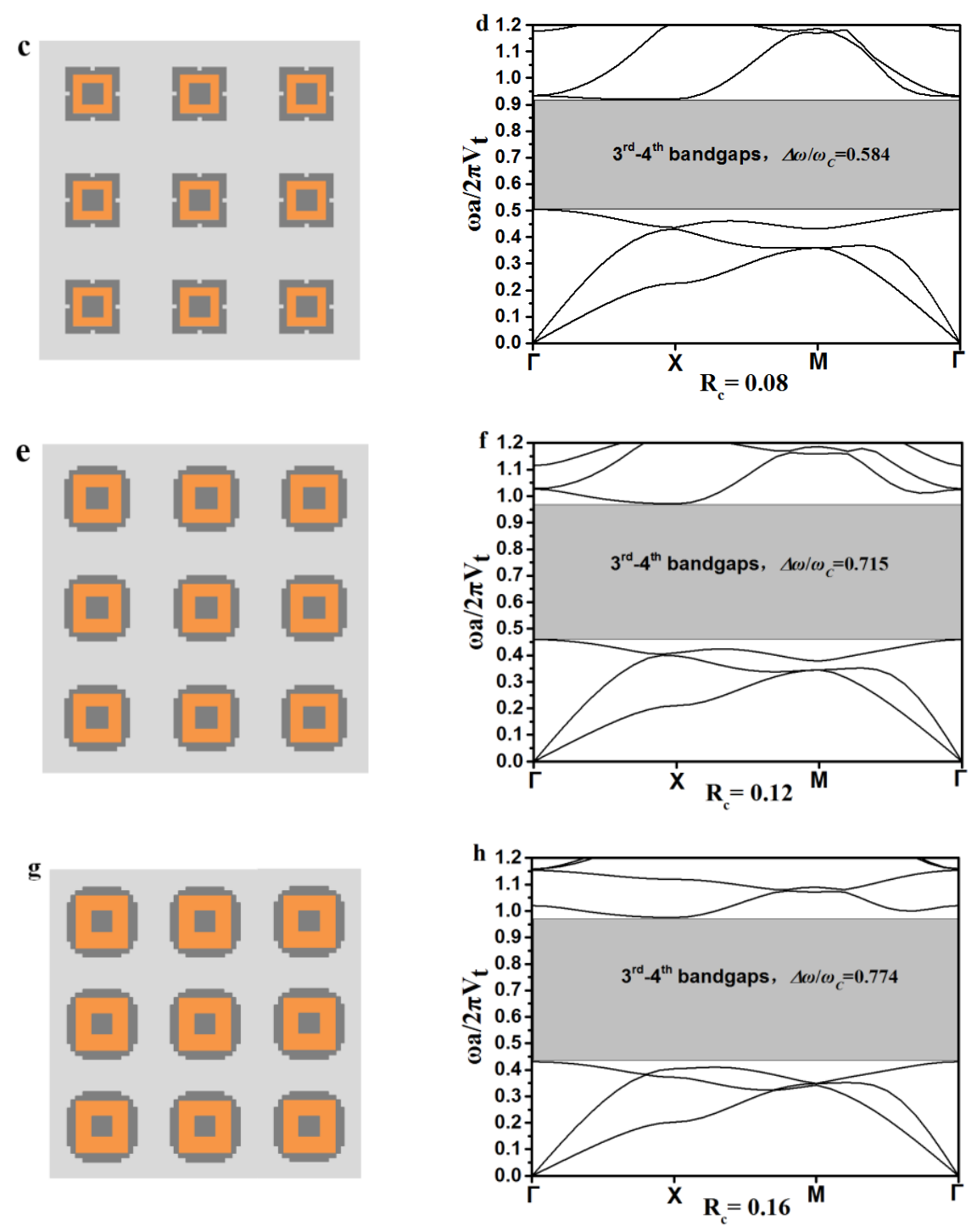

Fig.6. Optimized structures of $\mathrm{Pb}-\mathrm{Au} \mathrm{PnCs}$ and the corresponding band structures for the $3^{\text {rd }}-4^{\text {th }} \mathrm{ABG}$ with different Rc.

In order to see clearly the variation of $A B G$ with respect to $R_{c}$ in different systems, Fig. 7 plots the variation of $\mathrm{ABG}$ bandwidth with respect to $\mathrm{R}_{c}$ in Pb-Epoxy, Au-Epoxy, and Pb-Au PnCs, respectively. As shown in Fig. 7a and $7 b$, isolation strip is shown by the black wireframe slash with grey shadow. The variation trends for upper and lower edges of the ABGs in Pb-Epoxy and Au-Epoxy PnCs are similar. Along with the increase of the isolation ratio $\mathrm{R}_{c}$, bandwidths of ABGs in Pb-Epoxy PnCs are decreased from 0.302 at $\mathrm{R}_{c}=0$ to zero at $\mathrm{R}_{c}=0.15$. In Au-Epoxy PnCs, the bandwidth is decreased from 0.298 at $\mathbf{R}_{c}=0$ to 0.018 at $\mathbf{R}_{c}=0.16$. The main reason for the narrowing of the 
bandwidth is the drop of the upper band edge. The effect of $\mathrm{R}_{c}$ on the lower band edge could be ignored. When the isolation area is filled with $\mathrm{Au}$ (black wireframe slash with orange shadow as shown in Fig. 7c), the bandwidth variation displays the opposite trend. It increases from 0.302 to 0.774 along with $\mathbf{R}_{c}$ increasing from 0 to 0.16 .
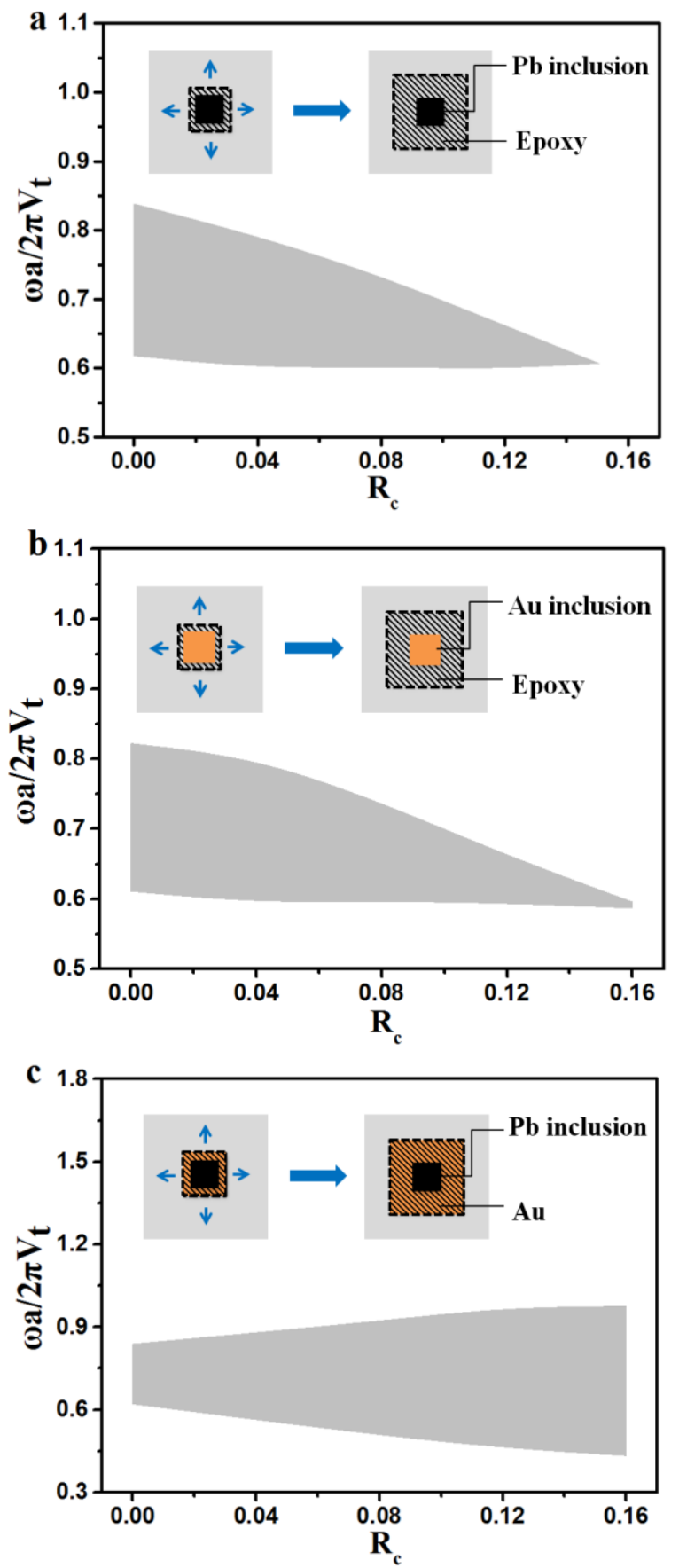

Fig. 7. Variation of upper and lower edges of the ABGs for (a) Pb-Epoxy PnCs, (b) Au-Epoxy PnCs and (c) Pb-Au PnCs. 


\section{Summary}

In this paper, a follow-up bandgap optimization for structures with reserved periodically distributed inclusions but no ABGs is proposed based on the BESO algorithm. The influence of inclusion and isolation strip on the optimized PnC structures is discussed in detail. Our results indicate that

1. Through proper topological optimization by adding filling materials, ABGs can be opened in a structure with periodically reserved inclusions to simultaneously achieve vibration suppression except for the functional demands; 2. Reserved inclusions display a material gravitation that the filling material will distribute around them in the optimization process. The higher the inclusion acoustic impedance, and the smaller the isolation strip are, the stronger this phenomenon is. In a system with periodically distributed pores, the material gravitation is not observed; 3. The material in isolation strip plays a decisive role on the optimized topology and ABG structures. When the acoustic impedance of isolation strip is smaller than that of inclusions, the upper band of the ABG is more sensitive to the expansion of isolation strip. Wider isolation strip will result in narrower ABG due to the dropping of the upper band; when the acoustic impedance of isolation strip is larger than that of inclusion, the ABG will become wider along with the isolation strip increasing, which is because in this situation the isolation strip enhances the material gravitation of inclusion, which is also observed in [2] .

The results in this paper provide a strategy to realize the multifunctional design in a structure with functional reserved inclusions. Our results also manifest the local resonance in the topology optimization, which is helpful in the acoustic design of active controlling PnCs.

\section{Acknowledgements}

The financial supports from the National Natural Science Foundation of China (No. 11772044) and the National 
Basic Research Program of China (973 Program) (No. 2015CB057800) are acknowledged.

[1] M.S. Kushwaha, P. Halevi, L. Dobrzynski, B. Djafari-Rouhani, Acoustic band structure of periodic elastic composites, Physical review letters, 71 (1993) 2022-2025.

[2] Z.Y. Liu, X.X. Zhang, Y.W. Mao, Y.Y. Zhu, Z.Y. Yang, C.T. Chan, P. Sheng, Locally resonant sonic materials, Science, 289 (2000) $1734-1736$.

[3] D.G. Zhao, W.G. Wang, Z.Y. Liu, J. Shi, W.J. Wen, Peculiar transmission property of acoustic waves in a one-dimensional layered phononic crystal, Physica B, 390 (2007) 159-166.

[4] Y.G. Li, T.N. Chen, X.P. Wang, S.B. Li, Lamb wave band gaps in one-dimensional radial phononic crystal plates with periodic double-sided corrugations, Physica B, 476 (2015) 82-87.

[5] Y.W. Yao, Z.L. Hou, F.G. Wu, X. Zhang, Low-frequency band gaps in one-dimensional thin phononic crystal plate with periodic stubbed surface, Physica B, 406 (2011) 2249-2253.

[6] A.L. Chen, Y.S. Wang, Study on band gaps of elastic waves propagating in one-dimensional disordered phononic crystals, Physica B, 392 (2007) 369-378.

[7] Z.S. Chen, Y.M. Yang, Z.M. Lu, Y.T. Luo, Broadband characteristics of vibration energy harvesting using one-dimensional phononic piezoelectric cantilever beams, Physica B, 410 (2013) 5-12.

[8] E.L. Albuquerque, P.D. Sesion, Band gaps of acoustic waves propagating in a solid/liquid phononic Fibonacci structure, Physica B, 405 (2010) 3704-3708.

[9] J.K. Huang, Z.F. Shi, W.X. Huang, Multiple band gaps of phononic crystals with quasi-Sierpinski carpet unit cells, Physica B, 516 (2017) 48-54.

[10] Y.G. Li, T.N. Chen, X.P. Wang, K.P. Yu, R.F. Song, Band structures in two-dimensional phononic crystals with periodic Jerusalem cross slot, Physica B, 456 (2015) 261-266.

[11] Y.S. Li, J.J. Chen, X. Han, K. Huang, J.G. Peng, Large complete band gap in two-dimensional phononic crystal slabs with elliptic 
inclusions, Physica B, 407 (2012) 1191-1195.

[12] X.J. Liu, Y.H. Fan, Y.M. An, The influence of T-square fractal shape holes on the band structure of two-dimensional phononic crystals, Physica B, 429 (2013) 73-78.

[13] A. Moghadasi, M.R. Roknabadi, S.R. Ghorbani, M. Modarresi, Electronic and phononic modulation of MoS2 under biaxial strain, Physica B, 526 (2017) 96-101.

[14] X.L. Zhou, C.Q. Chen, Tuning the locally resonant phononic band structures of two-dimensional periodic electroactive composites, Physica B, 431 (2013) 23-31.

[15] R. Sainidou, N. Stefanou, A. Modinos, Formation of absolute frequency gaps in three-dimensional solid phononic crystals, Phys Rev B, 66 (2002).

[16] L. Yan, H.P. Zhao, X.Y. Wang, G.S. Huang, X.Y. Peng, Band structure of three-dimensional phononic crystals, Chinese J Chem Phys, 19 (2006) 155-158.

[17] Z.Q. Zhan, P.J. Wei, Band Gaps of Three-Dimensional Phononic Crystal with Anisotropic Spheres, Mech Adv Mater Struc, 21 (2014) 245-254.

[18] X. Zhang, Z.Y. Liu, Y.Y. Liu, F.G. Wu, Elastic wave band gaps for three-dimensional phononic crystals with two structural units, Phys Lett A, 313 (2003) 455-460.

[19] Y.H. Liu, C.C. Chang, R.L. Chern, C.C. Chang, Phononic band gaps of elastic periodic structures: A homogenization theory study, Phys Rev B, 75 (2007).

[20] M.A. Attarzadeh, M. Nouh, Non-reciprocal elastic wave propagation in 2D phononic membranes with spatiotemporally varying material properties, Journal of Sound and Vibration, 422 (2018) 264-277.

[21] M.S. Kushwaha, P. Halevi, G. Martinez, L. Dobrzynski, B. Djafarirouhani, Theory of Acoustic Band-Structure of Periodic Elastic Composites, Phys Rev B, 49 (1994) 2313-2322.

[22] M.M. Sigalas, E.N. Economou, Acoustic Band-Structure of Periodic Elastic Composites - Comment, Physical review letters, 75 
(1995) 3580-3580.

[23] N.S. Gao, J.H. Wu, L. Jing, Research on the band gaps of the two-dimensional Sierpinski fractal phononic crystals, Mod Phys Lett B, 29 (2015).

[24] K. Wang, Y. Liu, T. Liang, B. Wang, Band structures in fractal grading porous phononic crystals, Journal of Physics and Chemistry of Solids, 116 (2018) 367-374.

[25] K. Wang, Y. Liu, T.S. Liang, Band structures in Sierpinski triangle fractal porous phononic crystals, Physica B, 498 (2016) $33-42$.

[26] O. Sigmund, J.S. Jensen, Systematic design of phononic band-gap materials and structures by topology optimization, Philos T Roy Soc A, 361 (2003) 1001-1019.

[27] M.I. Hussein, K. Hamza, G.M. Hulbert, R.A. Scott, K. Saitou, Multiobjective evolutionary optimization of periodic layered materials for desired wave dispersion characteristics, Structural and Multidisciplinary Optimization, 31 (2006) 60-75.

[28] H.W. Dong, X.X. Su, Y.S. Wang, C.Z. Zhang, Topological optimization of two-dimensional phononic crystals based on the finite element method and genetic algorithm, Structural and Multidisciplinary Optimization, 50 (2014) 593-604.

[29] Y.f. Li, X. Huang, F. Meng, S. Zhou, Evolutionary topological design for phononic band gap crystals, Structural and Multidisciplinary Optimization, 54 (2016) 595-617.

[30] Y.F. Li, F. Meng, S. Li, B.H. Jia, S.W. Zhou, X.D. Huang, Designing broad phononic band gaps for in-plane modes, Phys Lett A, 382 (2018) 679-684.

[31] Z.X. Zhang, Y.F. Li, F. Meng, X.D. Huang, Topological design of phononic band gap crystals with sixfold symmetric hexagonal lattice, Comp Mater Sci, 139 (2017) 97-105.

[32] Y.F. Li, X.D. Huang, S.W. Zhou, Topological Design of Cellular Phononic Band Gap Crystals, Materials, 9 (2016).

[33] L. Brillouin, Wave propagation in periodic structures: electric filters and crystal lattices, Courier Corporation, 2003.

[34] X. Huang, M. Xie, Evolutionary topology optimization of continuum structures: methods and applications, John Wiley \& Sons, 2010. 
[35] X. Huang, Y.M. Xie, Convergent and mesh-independent solutions for the bi-directional evolutionary structural optimization method,

Finite Elem Anal Des, 43 (2007) 1039-1049. 